\title{
Efeito colateral da pandemia de Covid-19 no Brasil sobre o número de procedimentos diagnósticos e de tratamento da sífilis
}

\author{
Tiago de Oliveira Furlam* \\ Claudia Cristina de Aguiar Pereira** \\ Gustavo Saraiva Frio ${ }^{\star \star *}$ \\ Carla Jorge Machado ${ }^{\star \star \star \star}$
}

\begin{abstract}
A sífilis, uma infecção vertical e sexualmente transmissível, curável e prevenível, é um problema de saúde pública no Brasil. Métodos diagnósticos e tratamentos são importantes no controle da doença. A pandemia de Covid-19 causou atrasos em diagnósticos e no tratamento na atenção primária em várias doenças e em diversos países, pois interrompeu padrões usuais de atendimento à saúde. 0 objetivo do estudo é identificar se houve menor número de procedimentos diagnósticos e de tratamento realizados para sífilis nos primeiros sete meses de 2020, comparativamente à média dos mesmos meses entre 2016 e 2019, no Brasil e nas unidades federativas. A redução no número de procedimentos seria um indicativo de atraso no diagnóstico, na detecção e no tratamento da sífilis em 2020. Foram utilizadas informações disponibilizadas no Sistema de Informações Ambulatoriais (SIA/SUS). Os achados para o Brasil indicaram queda de $1 / 3$ nos procedimentos de diagnóstico e de tratamento referentes à sífilis nos sete primeiros meses do ano da pandemia, comparados com a média dos sete primeiros meses nos quatro anos anteriores (2016-2019). Indicadores mostram diferenças importantes por unidades da federação, apontando para maiores quedas proporcionais nos volumes de procedimentos no Norte e Nordeste, com ênfase nos estados do Maranhão, Roraima, Pará, Bahia, Rio Grande do Norte, Amazonas, Pernambuco e Amapá.
\end{abstract}

Palavras-chave: Epidemia pelo novo coronavírus 2019. Serviços de saúde. Sífilis.

\footnotetext{
* Universidade Federal de Minas Gerais (UFMG), Belo Horizonte-MG, Brasil (to.furlan@outlook.com; https://orcid.org/00000001-8826-4964).

** Escola Nacional de Saúde Pública Sergio Arouca, Fundação Oswaldo Cruz (Ensp/Fiocruz), Rio de Janeiro-RJ, Brasil (pereirac. claudia@gmail.com; https://orcid.org/0000-0003-1389-9214).

*** Companhia de Planejamento do Distrito Federal (Codeplan), Brasília-DF, Brasil (gustavo.frio@gmail.com; https://orcid. org/0000-0001-6453-312X).

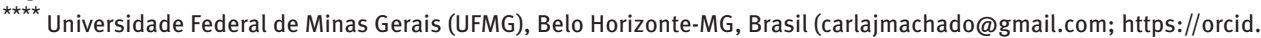
org/0000-0002-6871-0709).
} 


\section{Introdução}

A sífilis é uma infecção vertical e sexualmente transmissível (IST) que tem como agente etiológico a bactéria gram-negativa espiroqueta Treponema pallidum, da subespécie pallidum (ADAMS, 2021). Considera-se a infecção como sistêmica, crônica, curável e exclusiva do ser humano, com múltiplas manifestações de acordo com sua progressão temporal (BRASIL, 2020). A sífilis é um problema de saúde pública no Brasil e os métodos diagnósticos são de extrema importância para o manejo e o controle dessa questão em âmbitos individual e coletivo (BRASIL, 2019). A descrição clássica do sítio primário de infecção é a região genital, porém, outras áreas podem passar facilmente despercebidas devido à dificuldade de visualização da lesão, como a cérvice uterina, o ânus e o reto (MATTEl et al., 2012). Nesse cenário, em função da alta probabilidade de a doença não ser percebida no exame clínico isolado, há diversos testes diagnósticos que podem ser utilizados para detecção dessa infecção.

Exames diretos são os testes que realizam a pesquisa de T. pallidum em amostras coletadas diretamente das lesões sifilíticas (BRASIL, 2020). Já os testes sorológicos são os métodos mais utilizados na rotina clínica, úteis para o rastreio de indivíduos assintomáticos e para o diagnóstico de pacientes com sinais e sintomas sugestivos de sífilis (PEELING et al., 2017). Esses são divididos em testes não treponêmicos (TNT) e testes treponêmicos (TT). Os TNT são aqueles que mensuram imunoglobulinas (IgM e IgG) produzidas em resposta ao material liberado pelas bactérias e/ou pela célula hospedeira danificada pela infecção (PEELING et al., 2017). Além do uso para o diagnóstico, os TNT podem ser utilizados para o monitoramento da resposta ao tratamento e para o controle de cura da doença (BRASIL, 2020), visto que, após o tratamento, os títulos declinam e, na maioria dos indivíduos imunocompetentes, tornam-se não reativos em seis meses (PEELING et al., 2017). Já os TT detectam anticorpos específicos produzidos contra os antígenos de T. pallidum e podem ser úteis para detectar sífilis recente que passou despercebida aos TNT (PEELING et al., 2017). Os testes rápidos (TR), práticos e de fácil condução, que estão inclusos nos TT e são realizados com amostra de sangue colhida por punção digital ou venosa, podem ser feitos no momento da consulta e apresentam leitura rápida (resultado em no máximo 30 minutos) (BRASIL, 2020). Contudo, vale ressaltar que os TT têm a limitação de não servirem para distinguir infecção ativa de infecção passada e, portanto, não são úteis para avaliar a eficácia do tratamento (PEELING et al., 2017).

Há evidências de que a pandemia de Covid-19 muito provavelmente foi a causa de atrasos em diagnósticos na atenção primária, como indicado por um estudo do Reino Unido que utilizou como base os números de diagnósticos dos dez anos anteriores (WILLIAMS et al., 2020). Para CARR (2020), evidências referentes aos Estados Unidos mostram que a pandemia interrompeu os padrões usuais de atendimento à saúde, incluindo a suspensão temporária de alguns serviços médicos, especialmente a partir de março de 2020, levando a diagnósticos perdidos e atrasados. Ademais, para muitos, o medo do novo coronavírus 
transformou os estabelecimentos de saúde em locais de perigo, não de assistência (CARR, 2020). Com exceção das neoplasias malignas, houve queda acentuada e significativa de $43 \%$ a $50 \%$ nos diagnósticos das demais condições estudadas: transtornos mentais comuns, doenças do aparelho circulatório e diabetes tipo 2 (WILLIAMS et al., 2020). Para Williams et al. (2020), o número reduzido de novos diagnósticos observado no estudo reflete, provavelmente, um grande volume de casos verdadeiros de doenças que não foram detectadas, diagnosticadas ou tratadas, visto não haver justificativa aparente para tamanha queda na incidência real dessas condições. Para Czeisler et al. (2020), o atraso ou a evasão do atendimento aumenta a morbidade e o risco de mortalidade associado às doenças preveníveis, o que foi confirmado por pesquisa nos Estados Unidos, indicando que $40,9 \%$ dos entrevistados evitaram cuidados médicos durante a pandemia, sendo que mais de $30 \%$ destes postergaram os cuidados de rotina.

0 objetivo do presente estudo é identificar se houve menor número de procedimentos diagnósticos e de tratamento realizados para sífilis nos primeiros sete meses de 2020, comparativamente à média dos mesmos meses dos quatro anos anteriores (2016 a 2019). A redução no número de procedimentos seria um indicativo de atraso no diagnóstico, na detecção e no tratamento da sífilis em 2020, ano de início da pandemia de Covid-19. As análises foram feitas para o total de procedimentos no Brasil e também por unidade da federação, separadamente.

\section{Métodos}

Foram utilizados dados disponibilizados no Sistema de Informações Ambulatoriais (SIA/SUS) e incluídos os seguintes procedimentos no presente estudo: teste treponêmico para detecção de sífilis; teste não treponêmico para detecção de sífilis; teste anticorpo treponêmico fluorescente com absorção IgG (FTA-ABS IgG) para diagnóstico da sífilis; teste anticorpo treponêmico fluorescente com absorção IgM (FTA-ABS IgM) para diagnóstico da sífilis; testes rápidos para diagnóstico da sífilis; teste não treponêmico para detecção de sífilis em gestante; teste rápido para sífilis; teste rápido para sífilis na gestante ou pai/ parceiro; e administração de penicilina para tratamento da sífilis. As informações foram obtidas para janeiro a julho de 2016, 2017, 2018, 2019 e 2020, por unidade da federação. Foram feitas comparações entre o número médio de procedimentos dos referidos meses de 2016 a 2019 e o número de procedimentos no mesmo período de 2020. Além das análises por unidade da federação e para o total do Brasil, ao longo do tempo, os indicadores de interesse propostos foram:

- diferença entre o número de procedimentos diagnósticos e de tratamento realizados em 2020 comparativamente aos mesmos procedimentos nos quatro anos anteriores (média de 2016 a 2019), sendo que, em caso negativo, haveria indicativo de queda no diagnóstico, na detecção e no tratamento da sífilis durante o ano de início da 
pandemia de Covid-19. Estas análises foram feitas para o total do Brasil e por unidade da federação;

- diferença percentual, mês a mês, negativa e de magnitude superior a $100 \%$ entre os números de procedimentos de 2020 comparados com a média de 2016 a 2019 (total do Brasil e por unidade da federação);

- diferença percentual, mês a mês, negativa e de magnitude superior a $50 \%$ e não superior a $100 \%$ entre os números de procedimentos de 2020 comparados com a média de 2016 a 2019 (total do Brasil e por unidade da federação);

- proporção de diagnósticos em 2020 comparativamente à média de 2016-2019, por unidade da federação e para o Brasil, para todos os meses conjuntamente. Apenas para o Brasil essa análise foi realizada mensalmente.

O software utilizado para a elaboração dos gráficos foi o Microsoft Excel 11.0 for Mac.

\section{Resultados}

No Brasil, o número de procedimentos relacionados ao diagnóstico e tratamento de sífilis, entre 2016 e 2019, nos sete primeiros meses do ano, foi em média de 8,13 milhões (8.131.861), enquanto em 2020 correspondeu a 5,48 milhões (5.481.654) (Tabela 1). Portanto, nesse último ano houve 2,65 milhões de procedimentos a menos (2.650.207) em comparação ao período anterior. Em janeiro e julho de 2016-2019, os números foram, respectivamente, de 1,03 e 1,12 milhão. 0 número médio de procedimentos nesse período atingiu seu valor máximo em maio (1,41 milhão). Já em 2020, em janeiro e julho os números de procedimentos foram, respectivamente, 1,15 milhão (o maior volume de procedimentos nesse ano) e 690 mil (Tabela 1). Observa-se ainda que, entre janeiro e maio, as diferenças passaram de positivas (130 mil procedimentos a mais em janeiro de 2020 em relação à média de janeiro 2016-2019) a crescentemente negativas (800 mil a menos na comparação de maio 2016-2019) (Tabela 1). Em junho e julho, as diferenças permaneceram negativas, embora menos acentuadas (-570 mil e -430 mil, respectivamente).

TABELA 1

Número de procedimentos diagnósticos e de tratamento de sífilis Brasil - jan.-jul. 2016-2019 (média)-jan.-jul. 2020

\begin{tabular}{rlrrrrrrr}
\hline UF & & Janeiro & Fevereiro & \multicolumn{1}{c}{ Março } & \multicolumn{1}{c}{ Abril } & \multicolumn{1}{c}{ Maio } & \multicolumn{1}{c}{ Junho } & \multicolumn{1}{c}{ Julho } \\
\hline RO & $\begin{array}{l}\text { 2016-2019 } \\
\text { (média) }\end{array}$ & 11.588 & 11.912 & 12.715 & 12.340 & 12.704 & 12.178 & 14.786 \\
& & & & & & & & \\
& 2020 & 15.240 & 12.467 & 11.235 & 8.222 & 7.967 & 7.424 & 8.204 \\
& Diferença & 3.653 & 556 & -1.480 & $-4.118^{\star}$ & $-4.737^{\star}$ & $-4.754^{\star}$ & $-6.582^{\star}$ \\
\hline AC & $\begin{array}{l}\text { 2016-2019 } \\
\text { (média) }\end{array}$ & 5.235 & 5.096 & 5.312 & 5.921 & 7.587 & 6.455 & 6.207 \\
& 2020 & 7.186 & 6.839 & 5.505 & 4.135 & 3162 & 4.473 & 4.459 \\
& Diferença & 1.951 & 1.743 & 193 & -1.786 & $-4.425^{\star \star}$ & -1.982 & -1.748 \\
\hline
\end{tabular}


(continuação)

\begin{tabular}{|c|c|c|c|c|c|c|c|c|}
\hline UF & & Janeiro & Fevereiro & Março & Abril & Maio & Junho & Julho \\
\hline \multirow[t]{3}{*}{ AM } & $\begin{array}{l}\text { 2016-2019 } \\
\text { (média) }\end{array}$ & 39.865 & 71.308 & 41.860 & 38.145 & 41.963 & 37.918 & 38.634 \\
\hline & 2020 & 39.814 & 31.855 & 33.874 & 13.855 & 16.609 & 23.975 & 28.057 \\
\hline & Diferença & -51 & $-39.453^{\star \star}$ & -7.986 & $-24.290^{\star \star}$ & $-25.354^{\star \star}$ & $-13.943^{*}$ & -10.577 \\
\hline \multirow[t]{3}{*}{ RR } & $\begin{array}{l}\text { 2016-2019 } \\
\text { (média) }\end{array}$ & 2.079 & 1.964 & 2.307 & 1.893 & 2.085 & 2.059 & 2.110 \\
\hline & 2020 & 1.105 & 828 & 1.179 & 453 & 385 & 952 & 672 \\
\hline & Diferença & $-974^{*}$ & $-1.136^{\star \star}$ & $-1.128^{\star}$ & $-1.440^{\star \star}$ & $-1.700^{\star \star}$ & $-1.107^{\star \star}$ & $-1.438^{\star \star}$ \\
\hline \multirow[t]{3}{*}{ PA } & $\begin{array}{l}\text { 2016-2019 } \\
\text { (média) }\end{array}$ & 46.695 & 95.366 & 51.409 & 50.232 & 86.820 & 282.163 & 50.070 \\
\hline & 2020 & 50.146 & 50.996 & 46.303 & 37.884 & 31.275 & 32.362 & 26.941 \\
\hline & Diferença & 3.452 & $-44.370^{*}$ & -5.106 & -12.348 & $-55.545^{\star *}$ & $-249.801^{\star *}$ & $-23.129^{*}$ \\
\hline \multirow[t]{3}{*}{ AP } & $\begin{array}{l}\text { 2016-2019 } \\
\text { (média) }\end{array}$ & 3.019 & 3.340 & 3.682 & 3.428 & 3.664 & 2.802 & 2.642 \\
\hline & 2020 & 3.798 & 3.423 & 2.960 & 715 & 1.007 & 1.205 & 1.065 \\
\hline & Diferença & 779 & 84 & -722 & $-2.713^{\star \star}$ & $-2.657^{\star \star}$ & $-1.597^{\star \star}$ & $-1.577^{\star \star}$ \\
\hline \multirow[t]{3}{*}{ TO } & $\begin{array}{l}\text { 2016-2019 } \\
\text { (média) }\end{array}$ & 6.470 & 6.528 & 6.624 & 6.762 & 7.141 & 6.503 & 6.395 \\
\hline & 2020 & 7.207 & 7.871 & 7.666 & 5.241 & 5.796 & 5.519 & 5.616 \\
\hline & Diferença & 738 & 1.344 & 1.042 & -1.521 & -1345 & -984 & -779 \\
\hline \multirow[t]{3}{*}{ MA } & $\begin{array}{l}\text { 2016-2019 } \\
\text { (média) }\end{array}$ & 50.324 & 49.210 & 52.316 & 52.888 & 183.441 & 130.141 & 134.359 \\
\hline & 2020 & 48.189 & 51.256 & 44.731 & 27.376 & 26.091 & 26.168 & 22.669 \\
\hline & Diferença & -2.135 & 2.046 & -7.585 & $-25.512^{\star}$ & $-157.350 * *$ & $-103.973 * \star$ & $-111.690 * *$ \\
\hline \multirow[t]{3}{*}{$\mathrm{PI}$} & $\begin{array}{l}\text { 2016-2019 } \\
\text { (média) }\end{array}$ & 15.592 & 13.398 & 15.680 & 14.577 & 14.746 & 13.706 & 13.891 \\
\hline & 2020 & 14.840 & 14.215 & 12.393 & 5.505 & 7.642 & 9.037 & 8.501 \\
\hline & Diferença & -752 & 817 & -3.287 & $-9.072^{\star \star}$ & $-7.104^{*}$ & $-4.669^{\star}$ & $-5.390^{\star}$ \\
\hline \multirow[t]{3}{*}{ CE } & $\begin{array}{l}\text { 2016-2019 } \\
\text { (média) }\end{array}$ & 20.154 & 20.259 & 20.598 & 19.916 & 22.606 & 46.912 & 22.140 \\
\hline & 2020 & 25.734 & 24.297 & 18.733 & 13.256 & 12.500 & 16.213 & 14.291 \\
\hline & Diferença & 5.581 & 4.038 & -1.865 & $-6.660^{*}$ & $-10.106^{\star}$ & $-30.699^{\star \star}$ & $-7.849^{\star}$ \\
\hline \multirow[t]{3}{*}{ RN } & $\begin{array}{l}2016-2019 \\
\text { (média) }\end{array}$ & 12.231 & 13.002 & 12.952 & 12.380 & 16.850 & 12.985 & 13.267 \\
\hline & 2020 & 9.608 & 8.736 & 7.865 & 5.327 & 6.031 & 5.092 & 5.842 \\
\hline & diferença & -2.623 & -4.266 & $-5.087^{\star}$ & $-7.053^{\star \star}$ & $-10.819^{\star \star}$ & $-7.893^{\star \star}$ & $-7.425^{\star \star}$ \\
\hline \multirow[t]{3}{*}{ PB } & $\begin{array}{l}\text { 2016-2019 } \\
\text { (média) }\end{array}$ & 12.428 & 13.788 & 15.252 & 14.475 & 14.515 & 13.695 & 15.282 \\
\hline & 2020 & 23.776 & 15.323 & 10.891 & 8.037 & 6.758 & 7.609 & 11.623 \\
\hline & Diferença & 11.349 & 1.535 & -4.361 & $-6.438^{\star}$ & $-7.757^{\star \star}$ & $-6.086^{\star}$ & -3.659 \\
\hline \multirow[t]{3}{*}{ PE } & $\begin{array}{l}\text { 2016-2019 } \\
\text { (média) }\end{array}$ & 44.993 & 46.120 & 47.490 & 49.589 & 124.683 & 46.487 & 50.379 \\
\hline & 2020 & 53.978 & 48.040 & 41.641 & 23.557 & 24.084 & 26.646 & 30.939 \\
\hline & Diferença & 8.985 & 1.921 & -5.849 & $-26.032^{\star \star}$ & $-100.599^{\star \star}$ & $-19.841^{*}$ & $-19.440^{*}$ \\
\hline \multirow[t]{3}{*}{$\mathrm{AL}$} & $\begin{array}{l}\text { 2016-2019 } \\
\text { (média) }\end{array}$ & 11.423 & 11.981 & 13.378 & 12.145 & 13.508 & 12.244 & 14.802 \\
\hline & 2020 & 16.444 & 15.579 & 14.362 & 8.389 & 8.125 & 9.277 & 9.674 \\
\hline & Diferença & 5.021 & 3.598 & 984 & -3.756 & $-5.383^{*}$ & -2.967 & $-5.128^{\star}$ \\
\hline \multirow[t]{3}{*}{ SE } & $\begin{array}{l}\text { 2016-2019 } \\
\text { (média) }\end{array}$ & 8.084 & 7.820 & 10.061 & 8.881 & 9.500 & 7.978 & 9.890 \\
\hline & 2020 & 11.667 & 10.241 & 8.797 & 3.781 & 4.352 & 5.128 & 6.100 \\
\hline & Diferença & 3.583 & 2.421 & -1.264 & -5.100 ** & $-5.148^{\star \star}$ & $-2.850^{*}$ & $-3.790^{*}$ \\
\hline
\end{tabular}


(continuação)

\begin{tabular}{|c|c|c|c|c|c|c|c|c|}
\hline UF & & Janeiro & Fevereiro & Março & Abril & Maio & Junho & Julho \\
\hline \multirow[t]{3}{*}{ BA } & $\begin{array}{l}\text { 2016-2019 } \\
\text { (média) }\end{array}$ & 60.809 & 61.662 & 69.324 & 306.399 & 64.808 & 58.022 & 61.348 \\
\hline & 2020 & 56.442 & 58.168 & 52.602 & 40.727 & 42.648 & 46.776 & 45.478 \\
\hline & Diferença & -4.367 & -3.494 & -16.722 & $-265.672^{\star \star}$ & $-22.160^{\star}$ & -11.246 & -15.870 \\
\hline \multirow[t]{3}{*}{$M G$} & $\begin{array}{l}\text { 2016-2019 } \\
\text { (média) }\end{array}$ & 65.934 & 63.422 & 143.504 & 64.483 & 67.446 & 65.354 & 149.324 \\
\hline & 2020 & 203.146 & 73.783 & 60.883 & 42.511 & 46.801 & 48.544 & 48.878 \\
\hline & Diferença & 137.213 & 10.362 & $-82.621 * \star$ & $-21.972^{\star}$ & -20.645 & -16.810 & $-100.446^{\star \star}$ \\
\hline \multirow[t]{3}{*}{ ES } & $\begin{array}{l}\text { 2016-2019 } \\
\text { (média) }\end{array}$ & 22.547 & 21.359 & 24.438 & 24.151 & 24.854 & 22.994 & 25.623 \\
\hline & 2020 & 20.021 & 23.289 & 20.733 & 10.760 & 10.942 & 11.834 & 11.255 \\
\hline & Diferença & -2.526 & 1.930 & -3.705 & $-13.391^{\star \star}$ & $-13.912^{\star \star}$ & $-11.160^{*}$ & $-14.368^{\star \star}$ \\
\hline \multirow[t]{3}{*}{ RJ } & $\begin{array}{l}\text { 2016-2019 } \\
\text { (média) }\end{array}$ & 65.546 & 58.831 & 63.637 & 61.892 & 65.467 & 60.639 & 60.483 \\
\hline & 2020 & 69.104 & 57.241 & 56.743 & 39.548 & 43.677 & 45.255 & 48.804 \\
\hline & Diferença & 3.558 & -1.590 & -6.894 & $-22.344^{\star}$ & -21.790 & -15.384 & -11.679 \\
\hline \multirow[t]{3}{*}{$\mathrm{SP}$} & $\begin{array}{l}\text { 2016-2019 } \\
\text { (média) }\end{array}$ & 196.096 & 193.231 & 204.676 & 199.319 & 207.706 & 194.836 & 198.249 \\
\hline & 2020 & 261.724 & 230.741 & 209.270 & 134.853 & 152.086 & 180.919 & 199.990 \\
\hline & Diferença & 65.629 & 37.510 & 4.595 & -64.466 & -55.620 & -1.3917 & 1.741 \\
\hline \multirow[t]{3}{*}{ PR } & $\begin{array}{l}\text { 2016-2019 } \\
\text { (média) }\end{array}$ & 100.375 & 45.778 & 46.086 & 72.164 & 46.742 & 47.570 & 46.829 \\
\hline & 2020 & 48.047 & 45.813 & 49.121 & 35.206 & 38.920 & 39.659 & 36.400 \\
\hline & Diferença & $-52.328^{\star \star}$ & 35 & 3.036 & $-36.958^{\star *}$ & -7.822 & -7.911 & -10.429 \\
\hline \multirow[t]{3}{*}{$\mathrm{SC}$} & $\begin{array}{l}\text { 2016-2019 } \\
\text { (média) }\end{array}$ & 29.508 & 33.205 & 35.005 & 33.882 & 34.721 & 33.182 & 37.493 \\
\hline & 2020 & 36.870 & 37.619 & 30.384 & 21.247 & 26.018 & 27.664 & 24.350 \\
\hline & Diferença & 7.362 & 4.414 & -4.621 & $-12.635^{\star}$ & -8.703 & -5.518 & $-13.143^{*}$ \\
\hline \multirow[t]{3}{*}{ RS } & $\begin{array}{l}\text { 2016-2019 } \\
\text { (média) }\end{array}$ & 118.576 & 40.463 & 46.293 & 43.747 & 257.300 & 43.672 & 45.334 \\
\hline & 2020 & 49.170 & 43.110 & 41.400 & 28.280 & 31.782 & 33.753 & 33.537 \\
\hline & diferença & $-69.406^{\star \star}$ & 2.647 & -4.893 & $-15.467^{\star}$ & $-225.518^{\star \star}$ & -9.919 & -11.797 \\
\hline \multirow[t]{3}{*}{ MS } & $\begin{array}{l}\text { 2016-2019 } \\
\text { (média) }\end{array}$ & 13.624 & 141.046 & 16.017 & 15.371 & 15.174 & 14.593 & 15.619 \\
\hline & 2020 & 14.185 & 14.455 & 12.750 & 8.365 & 10.441 & 10.481 & 14.167 \\
\hline & Diferença & 561 & $-126.591^{\star \star}$ & -3.267 & $-7.006^{\star}$ & -4.733 & -4.112 & -1.452 \\
\hline \multirow[t]{3}{*}{ MT } & $\begin{array}{l}\text { 2016-2019 } \\
\text { (média) }\end{array}$ & 27.967 & 24.307 & 43.550 & 23660 & 24.795 & 27.348 & 49.744 \\
\hline & 2020 & 24.258 & 21.459 & 19.536 & 13992 & 16.852 & 15.884 & 13.277 \\
\hline & Diferença & -3.709 & -2.848 & $-24.014^{\star \star}$ & $-9.668^{\star}$ & -7.943 & $-11.464^{\star}$ & $-36.467^{\star \star}$ \\
\hline \multirow[t]{3}{*}{$\mathrm{GO}$} & $\begin{array}{l}\text { 2016-2019 } \\
\text { (média) }\end{array}$ & 24.827 & 24.506 & 56.702 & 24.886 & 25.761 & 25.056 & 25.259 \\
\hline & 2020 & 27.871 & 26.942 & 23.641 & 19.235 & 21.111 & 18.703 & 18.600 \\
\hline & Diferença & 3.044 & 2.437 & $-33.061^{\star \star}$ & -5.651 & -4.650 & -6.353 & -6.659 \\
\hline \multirow[t]{3}{*}{ DF } & $\begin{array}{l}\text { 2016-2019 } \\
\text { (média) }\end{array}$ & 8.931 & 8.900 & 10.520 & 11.025 & 10.153 & 10.052 & 8.783 \\
\hline & 2020 & 13.025 & 7.809 & 10.462 & 5.322 & 6.743 & 9.301 & 6.178 \\
\hline & Diferença & 4.094 & -1.091 & -58 & $-5703^{*}$ & $-3.410^{\star \star}$ & -751 & -2.605 \\
\hline \multirow[t]{3}{*}{ BRASIL } & $\begin{array}{l}\text { 2016-2019 } \\
\text { (média) }\end{array}$ & 1.024 .915 & 1.087 .796 & 1.071 .386 & 1.184 .546 & 1.406 .740 & 1.237 .540 & 1.118 .938 \\
\hline & 2020 & 1.152 .595 & 942.395 & 855.660 & 565.779 & 609.805 & 669.853 & 685.567 \\
\hline & Diferença & 127.680 & -145.401 & -215.726 & $-618.767^{\star \star}$ & $-796.935^{\star \star}$ & $-567.687^{\star}$ & $-433.371^{\star}$ \\
\hline
\end{tabular}

Fonte: Sistema de Informações Ambulatoriais (SIA/SUS). Elaboração dos autores.

* Diferença percentual negativa superior a $50 \%$ e não superior a $100 \%$. ** Diferença percentual negativa superior a $100 \%$. 
Quando comparado à média dos quatro anos anteriores (2016-2019), o total de procedimentos realizados no Brasil em janeiro de 2020 foi de $112 \%$, ou seja, superou em $12 \%$ a média do mesmo mês de anos anteriores (Gráfico 1). A partir de fevereiro de 2020 , houve decréscimo sustentado desse percentual, sendo que, nesse mês, os procedimentos corresponderam a $87 \%$ da média de fevereiro dos anos anteriores, enquanto em maio esse valor foi de $43 \%$, ou seja, $57 \%$ inferior à média do mesmo mês de anos anteriores. No conjunto dos meses analisados, os procedimentos em 2020 representaram $67 \%$ da média de anos anteriores, ou seja, 33\% inferiores (Gráfico 2).

GRÁFICO 1

Total de procedimentos diagnósticos e de tratamento de sífilis em 2020 como proporção da média de 2016-2019

Brasil - Janeiro-julho

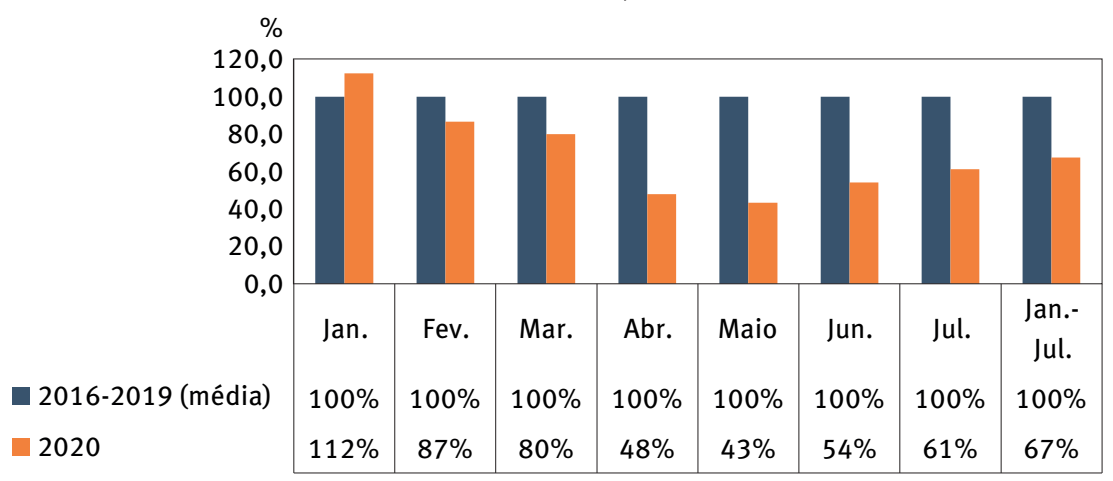

Fonte: Sistema de Informações Ambulatoriais (SIA/SUS). Elaboração dos autores.

Quanto às unidades da federação, podem ser indicadas semelhanças e diferenças entre os percentuais de procedimentos gerais em 2020 em relação à média dos quatro anos anteriores e ao nível do Brasil (Gráfico 2). Nenhum estado atingiu, em 2020, a média de procedimentos do período 2016-2019, sendo que aqueles que mais se aproximaram de $100 \%$ foram São Paulo (98\%), Tocantins (97\%) e Alagoas (91\%). É interessante observar que esses três estados localizam-se em três regiões distintas (Sudeste, Norte e Nordeste, respectivamente), indicando ausência de concentração em qualquer estado ou região do país. Três estados do Sudeste superaram a média do Brasil (67\%). Chama a atenção o fato de que alguns estados apresentaram menos da metade da média de procedimentos dos anos anteriores, dentre os quais Rio Grande do Sul (44\%), Pará (42\%), Roraima e Maranhão (ambos com 38\%) e Mato Grosso do Sul (37\%) (Gráfico 2). Mais uma vez, os estados relacionados encontram-se em distintas regiões do país: Sul, Norte, Nordeste e Centro-Oeste, respectivamente. 
GRÁFICO 2

Total de procedimentos diagnósticos e de tratamento de sífilis em 2020 como proporção da média de procedimentos diagnósticos e de tratamento em 2016-2019 Brasil - Janeiro-julho

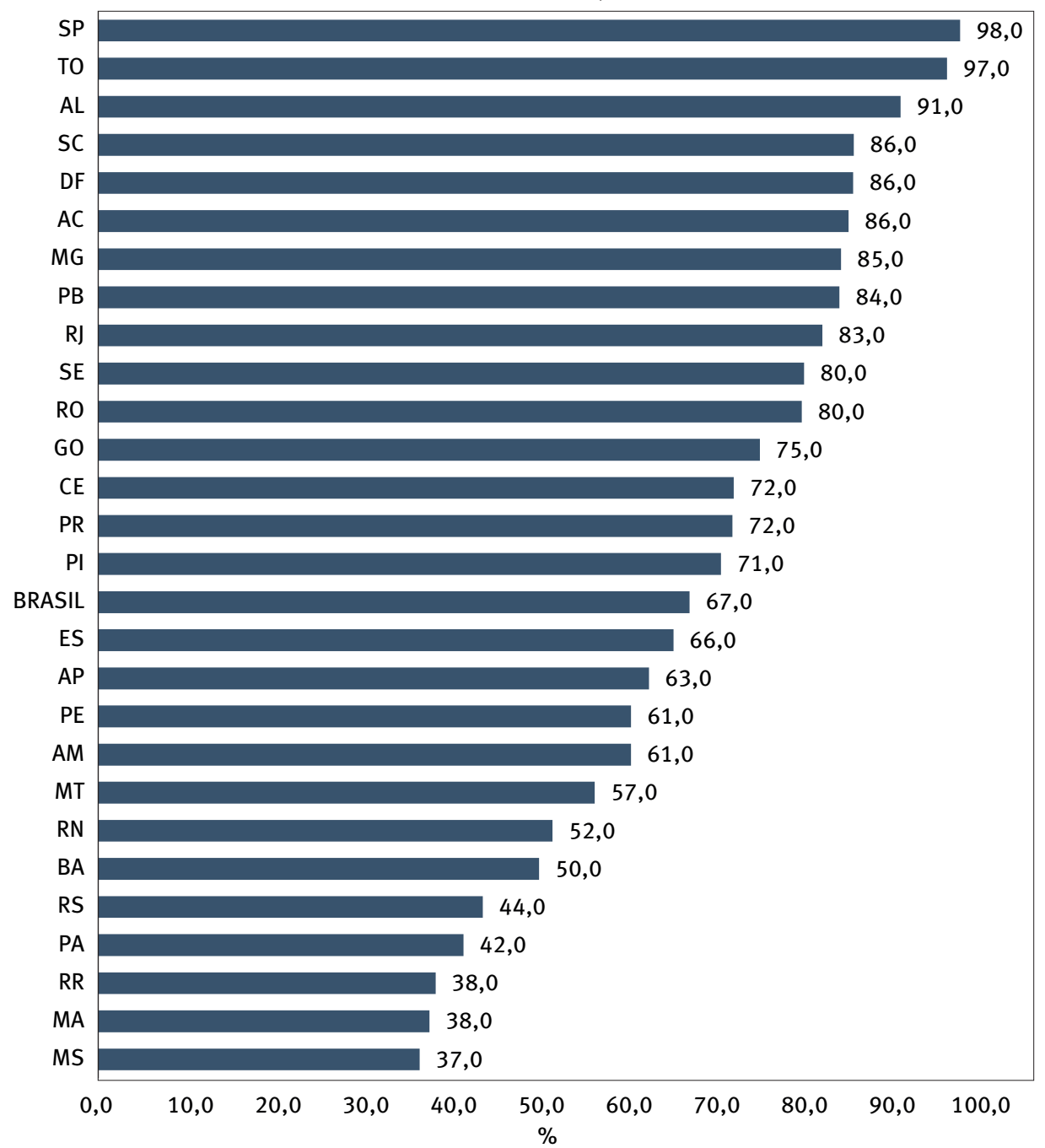

Fonte: Sistema de Informações Ambulatoriais (SIA/SUS). Elaboração dos autores.

Ainda tratando das unidades federativas, mas considerando a evolução temporal, em comparação às médias encontradas para os anos anteriores (2016-2019) nos mesmos meses, a região Norte apresentou diferentes cenários em 2020. Rondônia, Acre, Amapá e Tocantins iniciaram 2020 com quantidades de procedimentos superiores à média dos anos anteriores, coincidindo com o achado para o Brasil, enquanto Amazonas e Pará registraram valores muito semelhantes à média dos quatro anos prévios. Entretanto, em Roraima, o número de procedimentos foi menor do que a média dos outros anos já no mês de janeiro. Percebe-se, em seguida, comparativamente à média de 2016-2019, uma redução dos 
valores em fevereiro de 2020 no Amazonas, em Roraima e no Pará. Abril de 2020 foi o mês em que ocorreu uma tendência geral de redução dos procedimentos realizados frente aos anos anteriores, com pouca recuperação nos meses posteriores (Tabela 1), para todos os estados do Norte. Ou seja, ainda que pontos distintos de partida em janeiro de 2020 tenham existido entre os estados da região Norte, houve confluência para uma tendência comum geral, que foi a queda até abril de 2020 e manutenção dessa condição.

Ainda na região Norte, houve diferença percentual negativa e superior a $100 \%$ entre os números de procedimentos de 2020 comparados com a média de 2016 a 2019 (Tabela 1) no Acre (em maio), Amazonas (fevereiro, abril e maio), Roraima (todos os meses exceto janeiro e março), Pará (maio e junho) e Amapá (abril em diante). Já a diferença negativa no patamar superior a 50\%, mas não superior a $100 \%$, ocorreu em Rondônia (abril em diante), Amazonas (junho), Roraima (janeiro e março) e Pará (fevereiro e julho). Portanto, observa-se que 24 dos 49 (49\%) meses estudados no Norte tiveram diferenças percentuais negativas superiores a $50 \%$.

Quanto à região Nordeste, dos nove estados, cinco deles - Ceará, Paraíba, Pernambuco, Alagoas e Sergipe - iniciaram 2020 com quantidades de procedimentos superiores à média observada nos anos anteriores, enquanto Maranhão, Piauí e Bahia apresentaram quantidades de procedimentos similares à média dos anos passados. Já o Rio Grande do Norte teve número de procedimentos inferior à média passada. De janeiro a março, em comparação à média dos anos anteriores, o principal padrão observado foi de discreta queda no número de procedimentos em todos os estados, com exceção do Rio Grande do Norte, que registrou diferença percentual negativa entre $50 \%$ e $100 \%$ em março, e de Alagoas, que manteve um valor superior à média. A partir de março, todos os estados, com exceção de Alagoas, já apresentavam quantidades de procedimentos inferiores quando comparadas à média de 2016-2019. Assim como na região Norte, abril e maio são os meses em que a maioria dos estados registraram os menores números de procedimentos em 2020. A partir de maio, houve aparente tendência ao aumento discreto do número bruto de procedimentos, com exceção do Maranhão e Rio Grande do Norte. Contudo, nenhum estado recuperou a média dos anos anteriores.

Houve diferença percentual negativa e superior a 100\% (Tabela 1) nos seguintes estados do Nordeste: Maranhão (maio a julho), Piauí e Bahia (abril), Ceará (junho), Rio Grande do Norte (abril em diante), Paraíba (maio), Pernambuco (abril e maio) e Sergipe (abril e maio). Já as diferenças em níveis de 50\% a 100\% superiores e negativas ocorreram no Maranhão (abril), Piauí (maio em diante), Ceará (abril, maio e julho), Rio Grande do Norte (março), Paraíba (abril e junho), Pernambuco (junho e julho), Sergipe (junho e julho), Alagoas (maio e julho) e Bahia (maio). Assim, 32 dos 63 (50,8\%) meses estudados no Nordeste tiveram diferenças percentuais negativas superiores a $50 \%$. Os estados do Nordeste, portanto, em uma série de meses estudados, apresentaram déficits importantes quanto ao número de procedimentos realizados no período anterior de comparação.

No Sudeste, dos quatro estados, apenas o Espírito Santo iniciou o ano com número de procedimentos inferior à média de 2016-2019, sendo também o único que expressou 
aumento do número de procedimentos em fevereiro de 2020. A partir de março, todos os estados mantiveram uma tendência decrescente de procedimentos, chegando em abril com valores abaixo da média de 2016-2019. Após esse mês, Minas Gerais e Espírito Santo apresentaram leve tendência ao aumento, com valores se elevando pouco, enquanto Rio de Janeiro e São Paulo registraram aumentos mais expressivos, com São Paulo sendo o único estado que, em julho, alcançou número de procedimentos superior à média dos outros anos. Quanto aos indicadores percentuais, Minas Gerais (março e julho) e Espírito Santo (abril, maio e julho) tiveram diferenças negativas superiores a $100 \%$. Já as diferenças negativas de $50 \%$ a $100 \%$ ocorreram em abril nos estados de Minas Gerais e Rio de Janeiro e, em junho, no Espírito Santo. Logo, diferenças negativas superiores a 50\% ocorreram em oito meses para os estados da região Sudeste (8/28; $28,6 \%)$.

Na região Sul, Paraná e Rio Grande do Sul iniciaram o ano com quantidade de procedimentos inferiores à média de 2016-2019. O Paraná apresentou número superior à média de 2016-2019 em fevereiro e março, porém, voltou a registrar números inferiores a partir de abril, enquanto o Rio Grande do Sul atingiu número superior à média dos anos anteriores apenas em fevereiro. Já Santa Catarina iniciou o ano com número superior de procedimentos em relação à média e mostrou leve aumento em fevereiro. Em seguida apresentou expressiva queda em março, alcançando o menor valor em abril, não havendo recuperação dos valores médios de anos anteriores até o final da análise. Quanto aos indicadores percentuais, Paraná (janeiro e abril) e Rio Grande do Sul (janeiro e maio) tiveram diferenças negativas superiores a $100 \%$. Já as diferenças negativas de $50 \%$ a $100 \%$ ocorreram em Santa Catarina (abril e julho) e Rio Grande do Sul (abril). Assim, sete dos 21 $(33,3 \%)$ meses estudados dos estados da região Sul apresentaram diferenças percentuais negativas superiores a $50 \%$.

Finalmente, quanto à região Centro-Oeste, no início de 2020, o número de procedimentos ficou muito próximo à média de 2016-2019 no Mato Grosso do Sul, inferior à média no Mato Grosso e superior em Goiás e no Distrito Federal. Nesta última unidade federativa, houve oscilação no número de procedimentos em 2020: ficou abaixo da média em fevereiro; voltou para próximo da média em março; e retornou a cair em abril, apresentando o menor valor registrado nesse período. Entre maio e junho, o Distrito Federal registrou aumento do número de procedimentos, mas ainda se manteve abaixo da média de 2016-2019 e, em julho, ocorreu nova queda. Mato Grosso do Sul manteve número de procedimentos inferior à média de 2016-2019 desde fevereiro, enquanto no Mato Grosso tal situação ocorreu a partir de janeiro e, em Goiás, a partir de março. No concernente aos indicadores percentuais, as diferenças negativas superiores a $100 \%$ foram verificadas no Mato Grosso do Sul (fevereiro), Mato Grosso (março e julho), Goiás (março) e Distrito Federal (maio). Já as diferenças negativas de $50 \%$ a $100 \%$ ocorreram em abril no Mato Grosso do Sul, no Mato Grosso e no Distrito Federal e, em junho, no Mato Grosso. Nesta região, portanto, em nove dos 28 (32\%) meses analisados houve diferenças percentuais negativas superiores a $50 \%$. 


\section{Discussão}

O presente estudo salientou o impacto da pandemia de Covid-19 na produção de procedimentos diagnósticos e de tratamento de sífilis no Sistema Único de Saúde no Brasil como um todo e nas unidades da federação, separadamente. 0 trabalho abordou os sete primeiros meses da pandemia, período no decorrer do qual houve queda no número médio de procedimentos voltados para a sífilis comparativamente ao período pré-pandêmico.

Para Carr (2020), o número de diagnósticos perdidos ou atrasados é um dano colateral que se constituiria em uma epidemia paralela, caracterizando "uma das histórias não contadas pela pandemia" (tradução nossa). De fato, os achados preliminares para o Brasil e unidades federativas indicam queda de $1 / 3$ nos procedimentos de diagnóstico e de tratamento referentes à sífilis nos sete primeiros meses do ano da pandemia de Covid-19, em comparação com os sete primeiros meses dos quatro anos anteriores (2016-2019). Indicadores mostram diferenças importantes por unidades da federação, apontando para maiores quedas proporcionais nos volumes de procedimentos no Norte e Nordeste, com ênfase para os estados do Maranhão, Roraima, Pará, Bahia, Rio Grande do Norte, Amazonas, Pernambuco e Amapá, nessa ordem. Alguns desses estados vêm passando também por dificuldades no controle da Covid-19, como o Amazonas (ORELLANA et al., 2020), de forma que os achados do atual estudo indicam que os desafios poderão ser muito mais severos. Na Austrália, um estudo realizado na cidade de Melbourne também identificou, durante o lockdown da pandemia, reduções dramáticas nos atendimentos para infecções sexualmente transmissíveis, principalmente nos casos de baixo risco e de rastreamento de assintomáticos (CHOW et al., 2020). Cenários semelhantes também foram identificados nos Estados Unidos e na Itália (CRANE et al., 2020; LATINI et al., 2020; SACCHELLI et al., 2020).

Para Meyer et al. (2020), o mais preocupante é que alguns indivíduos podem apresentar sinais ou sintomas precoces de uma doença, mas não buscar avaliação, tratamento ou exames complementares. De fato, atrasos no diagnóstico e no tratamento podem ter implicações para o aprofundamento da severidade de condições de saúde. No caso da infecção pelo Treponema pallidum, o paciente pode apresentar diversas manifestações, desde as lesões iniciais, na chamada sífilis primária, até, quando não tratada, quadros de sífilis terciária, que podem se manifestar após período variável de latência (1 a 40 anos após a infecção) (BRASIL, 2020). Assim, a perda da oportunidade de diagnóstico e tratamento precoce dessa doença pode trazer consequências a longo prazo, não se limitando somente ao ano da pandemia. Dessa forma, um aumento na demanda pode ser esperado nos períodos posteriores às fases mais críticas da pandemia (CHOW et al., 2020) e medidas devem ser planejadas para priorizar pacientes com diagnósticos tardios, o que é crucial para prevenir uma sobrecarga dos serviços de saúde no futuro.

Finalmente, cabe observar que a sífilis é uma doença prevenível e tratável e não deve ser negligenciada durante a pandemia de Covid-19 (DI BUDUO et al., 2020). A presente nota de pesquisa traz um alerta para além de números inferiores de procedimentos de 
diagnósticos e tratamentos de sífilis no país. Vale ressaltar que o pouco contato com os serviços de saúde também é preocupante, visto esse ser o local em que as pessoas têm acesso a ações de promoção de saúde e prevenção de doenças. 0 presente trabalho apresentou enfoque na sífilis, porém, o real dano da redução do número de procedimentos de diagnóstico e tratamento vai além dessa doença, sendo o verdadeiro impacto da pandemia ainda inestimável. Portanto, é necessário que novas pesquisas e mensurações sejam realizadas com o objetivo de avaliar a extensão dos danos e perdas que a pandemia de Covid-19 trouxe à saúde pública no Brasil e pode vir a trazer nos anos vindouros, de forma a possibilitar a elaboração de políticas públicas de saúde que sejam assertivas e efetivas.

\section{Referências}

ADAMS, D. Foundations of infectious diseases: a public health perspective. Burlington: Jones \& Bartlett Learning, 2021.

BRASIL. Ministério da Saúde. Secretaria de Vigilância em Saúde. Boletim Epidemiológico: Sífilis, número especial, out. 2019. Disponível em: https://portaldeboaspraticas.iff.fiocruz.br/ biblioteca/boletim-epidemiologico-sifilis-2019/. Acesso em: 9 fev. 2021.

BRASIL. Ministério da Saúde. Secretaria de Vigilância em Saúde. Protocolo clínico e diretrizes terapêuticas para atenção integral às pessoas com infecções sexualmente transmissíveis (IST). Brasília, 2020. Disponível em: http://www.aids.gov.br/pt-br/pub/2015/protocolo-clinico-ediretrizes-terapeuticas-para-atencao-integral-pessoas-com-infeccoes. Acesso em: 15 fev. 2021.

CARR, S. Missed and delayed diagnoses of non-COVID conditions - collateral harm from a pandemic. ImproveDx Newsletter, v. 7, n. 4, Jul. 2020. Disponível em: https://www. improvediagnosis.org/improvedx-newsletter/improvedx-july-2020/missed-and-delayeddiagnoses-of-non-covid-conditions-collateral-harm-from-a-pandemic/. Acesso em: 12 fev. 2021.

CHOW, E.; HOCKING, J.; ONG, J.; PHILLIPS, T.; FAIRLEY, C. Sexually transmitted infection diagnoses and access to a sexual health service before and after the national lockdown for COVID-19 in Melbourne, Australia. Open Forum Infectious Diseases, v. 8, n. 1, ofaa536, 2020. Disponível em: DOI: 10.1093/ofid/ofaa536. Acesso em: 14 fev. 2021.

CRANE, M.; POPOVIC, A.; STOLBACH, A.; GHANEM, K. Reporting of sexually transmitted infections during the COVID-19 pandemic. Sexually Transmitted Infections, v. 97, n. 2, p. 101-102, Nov. 2020. Disponível em: DOI: 10.1136/sextrans-2020-054805. Acesso em: 14 fev. 2021.

CZEISLER, M.; MARYNAK, K.; CLARKE, K. E. N.; SALAH, Z.; SHAKYA, I.; THIERRY, J. A. M.; ALI, N.; MCMILLAN, H.; WILEY, J. F.; WEAVER, M. D.; CZEISLER, C. A.; RAJARATNAM, S. M. W.; HOWARD, M. E. Delay or avoidance of medical care because of COVID-19 - Related Concerns - United States, June 2020. Morbidity and Mortality Weekly Report, v. 69, n. 36, 2020. Disponível em: https://doi.org/10.15585/mmwr.mm6936a4. Acesso em: 9 fev. 2021.

DI BUDUO, A.; ATZORI, L.; PILLONI, L.; PERLA, S.; RONGIOLETTI, F.; FERRELI, C. Don't forget about syphilis: sexually transmitted diseases during COVID-19 pandemic. Journal of Public Health Research, v. 9, n. 4, 2020. Disponível em: DOI: 10.4081/jphr.2020.2040. Acesso em: 14 fev. 2021.

LATINI, A.; MAGRI, F.; DONÀ, M. G.; GIULIANI, M.; CRISTAUDO, A.; ZACCARELLI, M. Is COVID-19 affecting the epidemiology of STIs? The experience of syphilis in Rome. Sexually Transmitted Infections, v. 97, n. 1, Jul. 2020. Disponivel em: DOl: https://doi.org/10.1136/ sextrans-2020-054543. Acesso em: 14 fev. 2021. 
MATTEI, P. L.; BEACHKOFSKY, T. M.; GILSON, R. T.; WISCO, O. J. Syphilis: a reemerging infection. American Family Physician, v. 86, n. 5, p. 433-440, 2012. Disponível em: https://www.aafp.org/ afp/2012/0901/p433.html. Acesso em: 14 fev. 2021.

MEYER, M.; BINDELGLAS, E.; KUPFERMAN, M. E.; EGGERMONT, A. M. The ongoing COVID-19 pandemic will create a disease surge among cancer patients. Ecancermedicalscience, v. 14, n. 105, 2020. Disponível em: https://ecancer.org/en/journal/editorial/105-the-ongoing-covid-19pandemic-will-create-a-disease-surge-among-cancer-patients/pdf. Acesso em: 12 fev. 2021.

ORELLANA, J. D. Y.; CUNHA, G. M.; MARRERO, L.; HORTA, B. L.; LEITE, I. C. Explosion in mortality in the Amazonian epicenter of the COVID-19 epidemic 19. Cadernos de Saúde Pública, v. 36, n. 7, e00120020, 2020. Disponivel em: http://www.scielo.br/scielo.php?script=sci_ arttext\&pid=S0102-311X2020000706001\&lng=en\&nrm=iso. Acesso em: 15 fev. 2021.

PEELING, R. W.; MABEY, D.; KAMB, M. L.; CHEN, X. S.; RADOLF, J. D.; BENZAKEN, A. S. Syphilis. Nature Reviews. Disease Primers, v. 3, 17073, 2017. Disponível em: https://doi.org/10.1038/ nrdp.2017.73. Acesso em: 12 fev. 2021.

SACCHELLI, L. et al. Sexually transmitted infections during the COVID-19 outbreak: comparison of patients referring to the service of sexually transmitted diseases during the sanitary emergency with those referring during the common practice. Journal of European Academy Dermatology and Venereology, v. 34, n. 10, p. e553-e556, 2020. Disponível em: https://doi.org/10.1111/jdv.16694. Acesso em: 14 fev. 2021.

WILLIAMS, R.; JENKINS, D. A.; ASHCROFT, D. M.; BROWN, B.; CAMPBELL, S.; CARR, M. J.; CHERAGHI-SOHI, S.; KAPUR, N.; THOMAS, O.; WEBB, R. T.; PEEK, N. Diagnosis of physical and mental health conditions in primary care during the COVID-19 pandemic: a retrospective cohort study. Lancet Public Health, v. 5, n. 10, p. e543-e550, 2020. Disponível em: https://www.ncbi. nlm.nih.gov/pmc/articles/PMC7511209/. Acesso em: 9 fev. 2021.

\section{Sobre os autores}

Tiago de Oliveira Furlam é acadêmico do $8^{\circ}$ período do curso de Medicina pela Faculdade de Medicina da Universidade Federal de Minas Gerais.

Claudia Cristina de Aguiar Pereira é PhD pela Universidade de Wisconsin e pesquisadora da Escola Nacional de Saúde Pública Sérgio Arouca, Fundação Oswaldo Cruz.

Gustavo Saraiva Frio é doutor em Economia pelo Programa de Pós-Graduação em Economia do Desenvolvimento da Pontifícia Universidade Católica do Rio Grande do Sul. Gerente de Pesquisas na Diretoria de Estudos e Políticas Sociais (Dipos) da Codeplan (Companhia de Planejamento do Distrito Federal).

Carla Jorge Machado é PhD pela Universidade Johns Hopkins e professora titular do Departamento de Medicina Preventiva e Social da Faculdade de Medicina da Universidade Federal de Minas Gerais.

\section{Endereço para correspondência}

Tiago de Oliveira Furlam

Rua Rio Grande do Norte, 888, Funcionários

30130-131 - Belo Horizonte-MG, Brasil 


\author{
Claudia Cristina de Aguiar Pereira \\ Escola Nacional de Saúde Pública Sérgio Arouca \\ Rua Leopoldo Bulhões, 1480, Manguinhos \\ 21041-210 - Rio de Janeiro-RJ, Brasil \\ Gustavo Saraiva Frio \\ Companhia de Planejamento do Distrito Federal \\ Setor de Administração Municipal - SAM, Bloco H, Setores Complementares \\ 70620-080 - Brasília-DF, Brasil \\ Carla Jorge Machado \\ Faculdade de Medicina da Universidade Federal de Minas Gerais \\ Avenida Professor Alfredo Balena, 190, Santa Efigênia \\ 30130-100 - Belo Horizonte-MG, Brasil
}

\title{
Abstract
}

Side effect of the COVID-19 pandemic in Brazil on the number of diagnostic and treatment procedures for syphilis

Syphilis, a vertical and sexually transmitted infection, curable and preventable, is a public health problem in Brazil. Diagnostic methods and treatments are important in controlling the disease. The COVID-19 pandemic caused delays in diagnosis and lack of treatment in primary care in several diseases and in several countries, as the pandemic disrupted usual health care standards. The aim of the study was to identify whether there were fewer diagnostic and treatment procedures performed for syphilis in the first seven months of 2020, compared to the average for the same months between 2016 and 2019, in Brazil and Federative Units. The reduction in the number of procedures would be indicative of a delay in the diagnosis, detection and treatment of syphilis in 2020. Information used came from the Outpatient Information System (SIA / SUS). The findings for Brazil indicated a 1/3 drop in diagnosis and treatment procedures for syphilis in the first seven months of the year of the COVID-19 pandemic, compared with the first seven months of the previous four years (2016-2019). Indicators showed important differences by Federation Units, pointing to greater proportional decrease in the volume of procedures in the North and Northeast, with an emphasis on Maranhão, Roraima, Pará, Bahia, Rio Grande do Norte, Amazonas, Pernambuco and Amapá.

Keywords: 2019-nCoV pandemic. Health services. Syphilis.

\section{Resumen}

Efecto secundario de la pandemia de Covid-19 en Brasil sobre el número de procedimientos de diagnóstico y tratamiento de la sífilis

La sífilis, una infección vertical y de transmisión sexual, curable y prevenible, es un problema de salud pública en Brasil. Los métodos de diagnóstico y los tratamientos son importantes para controlar la enfermedad. La pandemia de Covid-19 provocó retrasos en el diagnóstico y tratamiento en la atención primaria de variadas enfermedades en varios países, ya que interrumpió los estándares habituales de atención de la salud. El objetivo del estudio fue identificar si se realizaron menos procedimientos de diagnóstico y tratamiento de la sífilis en 
los primeros siete meses de 2020 en comparación con la media de los mismos meses entre 2016 y 2019, en Brasil y en sus unidades federativas. La reducción del número de procedimientos indicaría indicativo de un retraso en el diagnóstico, la detección temprana y el tratamiento de la sífilis en 2020. Para ello se utilizó la información disponible en el Sistema de Información Ambulatoria (SIA/SUS). Los hallazgos indicaron una caída de un tercio en los procedimientos de diagnóstico y tratamiento de la sífilis en los primeros siete meses del año de la pandemia de Covid-19 para Brasil, en comparación con los primeros siete meses de los cuatro años anteriores (2016-2019). Los indicadores mostraron diferencias importantes por unidades de la Federación, apuntando a mayores caídas proporcionales en el volumen de trámites en el Norte y Nordeste, con énfasis en Maranhão, Roraima, Pará, Bahia, Rio Grande do Norte, Amazonas, Pernambuco y Amapá.

Palabras clave: Epidemia por el nuevo coronavirus 2019. Servicios de salud. Sífilis.

Recebido para publicação em 16/02/2021 Aceito para publicação em 23/08/2021 